\title{
Retrospect and Prospect of Aircraft Comfort Design
}

\author{
Peng Li and Kaixiang Li
}

\begin{abstract}
Passenger comfort has not been completely integrated into the entire aircraft design process yet. Sometimes, the cabin vibration environment is intolerant during flight, causing seriously adverse reactions such as discomfort, fatigue, and reduced task-performing ability. Here, the aircraft digital FEM model is used to predict the cabin environment, and the acceleration response results can be used as the input to evaluate the human comfort level. Four methods for evaluating human comfort level (the absorbing power method, NASA ride quality method, ISO and BSI standard) are commonly used. The four methods are coded into a plug-in program, which can be used in aircraft FEM model, to facilitate the evaluation process. Finally, the procedures and suggestions of cabin comfort design are given, for example, engine damping installation, seat cushion updating, seat vibration isolation treatment, etc. In this way, the aircraft structure can be modified and improved according to the passenger comfort feedback.
\end{abstract}

P. Li $(\bowtie) \cdot \mathrm{K} . \mathrm{Li}$

Aircraft Strength Research Institute, Yanta District, 86 Dianzierlu Road, Xi' an Cty, China

(C) The Author(s), under exclusive license to Springer Nature Switzerland AG 2021 